\title{
SIMULATION AND DIAGNOSTICS OF PLASMACHEMICAL PROCESSES DURING MICROWAVE PLASMA SYNTHESIS OF GRAPHENE NANOSHEETS FROM ETHANOL
}

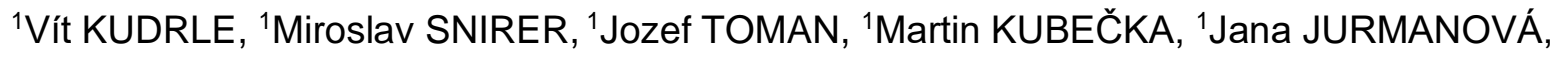 \\ ${ }^{1}$ Ondřej JAŠEK, ${ }^{2}$ František KRČMA \\ ${ }^{1}$ Department of Physical Electronics, Faculty of Science, Masaryk University, Brno, Czech Republic, EU, \\ kudrle@sci.muni.cz \\ 2Institute of Physical and Applied Chemistry, Brno University of Technology, Brno, Czech Republic, EU
}

https://doi.org/10.37904/nanocon.2020.3691

\begin{abstract}
Plasma synthesis by ethanol decomposition in microwave atmospheric torch is a simple, efficient, single-step scalable method suitable for volume production of graphene nanosheets. In our work, we studied influence of microwave power on several plasma parameters (e.g. gas temperature, concentration of active species) by optical emission spectroscopy (OES), Fourier transform infrared spectrometry (FTIR) and mass spectrometry (MS) to better understand the process of precursor decomposition and graphene formation in the gas phase. We observed significant change in kinetics and influence of input power on ethanol decomposition routes. Results were compared with theoretical model comprising hydrodynamics, plasma, heat transfer and chemical kinetics.
\end{abstract}

Keywords: Graphene, microwave plasma torch, OES, ethanol, modelling

\section{INTRODUCTION}

Atmospheric pressure plasma synthesis of advanced materials, including the graphene nanosheets, brings some advantages over competing methods e.g. liquid phase exfoliation and chemical vapour deposition. While commonly used, these techniques have their limitations in a need of a substrate or a post-treatment to obtain separated high-quality graphene. In contrast, a microwave plasma synthesis of graphene is a rather recent method $[1,2,3]$ based on decomposition of organic precursors which in many aspects overcomes these limitations. It is mostly single step, without all the complexities of the wet process, uses simple equipment and affordable precursors, etc. However, the inherent nonlinearities of plasma, its spatial inhomogeneities and nonequilibrium kinetics make the process critically dependent on optimal local conditions. This in turn makes the analysing, controlling and up-scaling quite a challenge.

Material analyses of the graphene product by e.g. electron microscopy (SEM, TEM) and/or Raman spectroscopy can be carried out for various experimental conditions. While they provide ultimate results about the product quality, the causes can remain hidden. The diagnostics of the synthesis process itself is thus needed. The optical emission spectroscopy (OES) provides localised information, but only on radiating species, However, the more complex organic molecules involved in graphene growth are often formed later, outside the active plasma itself. These can be detected by standard chemical gas analysis techniques, such as Fourier transform infrared (FTIR), gas chromatography (GC) and mass spectroscopy (MS) but these are ex-situ and cannot provide localised information.

In this paper we combine these diagnostic techniques with a theoretical model, which couples heat transfer, gas dynamics and chemical kinetics, to get deeper insight into plasmachemical processes during the graphene synthesis. 


\section{EXPERIMENTAL SET-UP}

The plasma torch was excited by a $2.45 \mathrm{GHz}$ microwave generator with a power variable in the 10-500 W range. The generator fed a microwave waveguide line ending with a transition to rigid coaxial transition. Its central conductor ( $6 \mathrm{~mm}$ diameter) was hollow which enabled passing of multiple gas lines inside. It ended in an exchangeable carbon nozzle with dual flow configuration enhancing the plasma stability [3]. The central (axial) channel $(0.8 \mathrm{~mm}$ diameter) was used for argon (300-1000 sccm). The precursor (ethanol vapour from a bubbler, 10-700 sccm argon carrier gas) is introduced to an outer (annular) channel, shown in Figure 1.

Plasma reactor consisted of fused silica tube $(80 \mathrm{~mm}$ diameter, $200 \mathrm{~mm}$ long) terminated by a duralumin flange. The exhaust gas samples from the T-piece were collected in transportable cuvette for later FTIR and GC analysis. Optical emission spectra were recorded through the reactor walls and magnifying optical system using Jobin Yvon Triax 550 spectrometer. Digital imaging was performed using intensified CCD Pi-MAX 3 camera equipped with $100 \mathrm{~mm}$ macro UV lens. Set of interference filters was used for spectral imaging. Deposited nanomaterial was collected from the reactor walls for further analysis. Graphene nanosheets were imaged with scanning electron microscope (SEM) TESCAN MIRA3 with Schottky field emission electron gun. Raman spectroscopy was carried out using the HORIBA LabRAM HR Evolution system with $532 \mathrm{~nm}$ laser, using $100 \mathrm{x}$ objective, $600 \mathrm{gr} / \mathrm{mm}$ grating and $30 \mathrm{~s}$ acquisition time in the range from 1000 to $3200 \mathrm{~cm}^{-1}$.

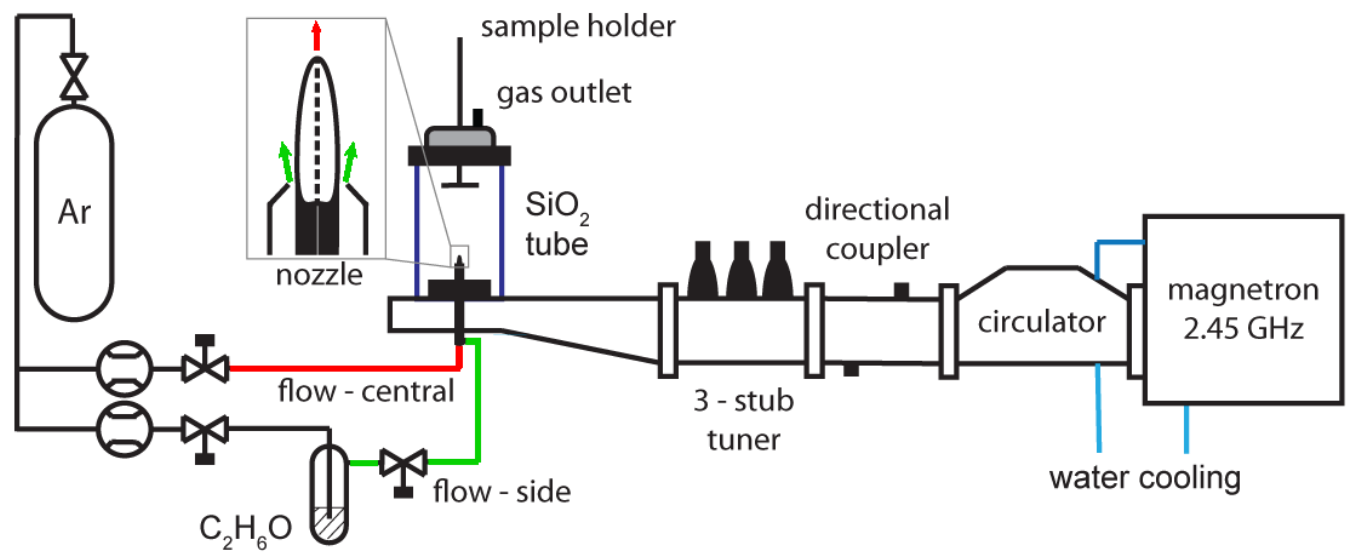

Figure 1 Experimental set-up

\section{THEORETICAL MODEL}

COMSOL Multiphysics was used for solving our model combining the gas dynamics, heat transfer and chemical reactions. Two-dimensional axially symmetric domain was used to simulate the geometry of our experimental reactor. The model takes the experimentally measured plasma plume dimensions as an input for the gas heat source. The gas flow is considered to be non-isotermal and turbulent. The main gas flow (500 sccm of argon) to the domain is introduced via the central (axial) channel, and the outer annular channel supplied $700 \mathrm{sccm}$ of argon with $10 \mathrm{sccm}$ of ethanol vapours. The reduced chemical reaction scheme of the ethanol decomposition was taken from A. Merino [4] and consists of 66 reactions and 31 species.

\section{RESULTS AND DISCUSSION}

The dual flow configuration, where the precursor was introduced into the outer channel while the active plasma itself was burning mostly in pure argon, proved to be very stable and efficient in ethanol conversion. The discharge had a form of stable, axially symmetric filament which length depended linearly on absorbed microwave power (behaviour typical for surface wave driven discharges [5]). Optical emission spectroscopy showed (Figure 2) that emissions were dominated by very intense molecular $\mathrm{C}_{2}$ Swan band system (maximum 
at $516 \mathrm{~nm}$ ). Despite the precautions (argon flushing, tightened seals, etc.), there were still traces of air in our apparatus, as exhibited by strong emissions of cyan violet system $\mathrm{CN}(\mathrm{B}-\mathrm{X})$ (maximum at $388 \mathrm{~nm}$ ). The atomic carbon $C$ was also detected at $247 \mathrm{~nm}$ but with rather small intensity. High intensity of $\mathrm{C}_{2}$ bands suggest higher concentrations of $\mathrm{C}_{2}$ which serves as precursor for graphene formation while atomic $\mathrm{C}$ often leads to formation of amorphous structures [6].

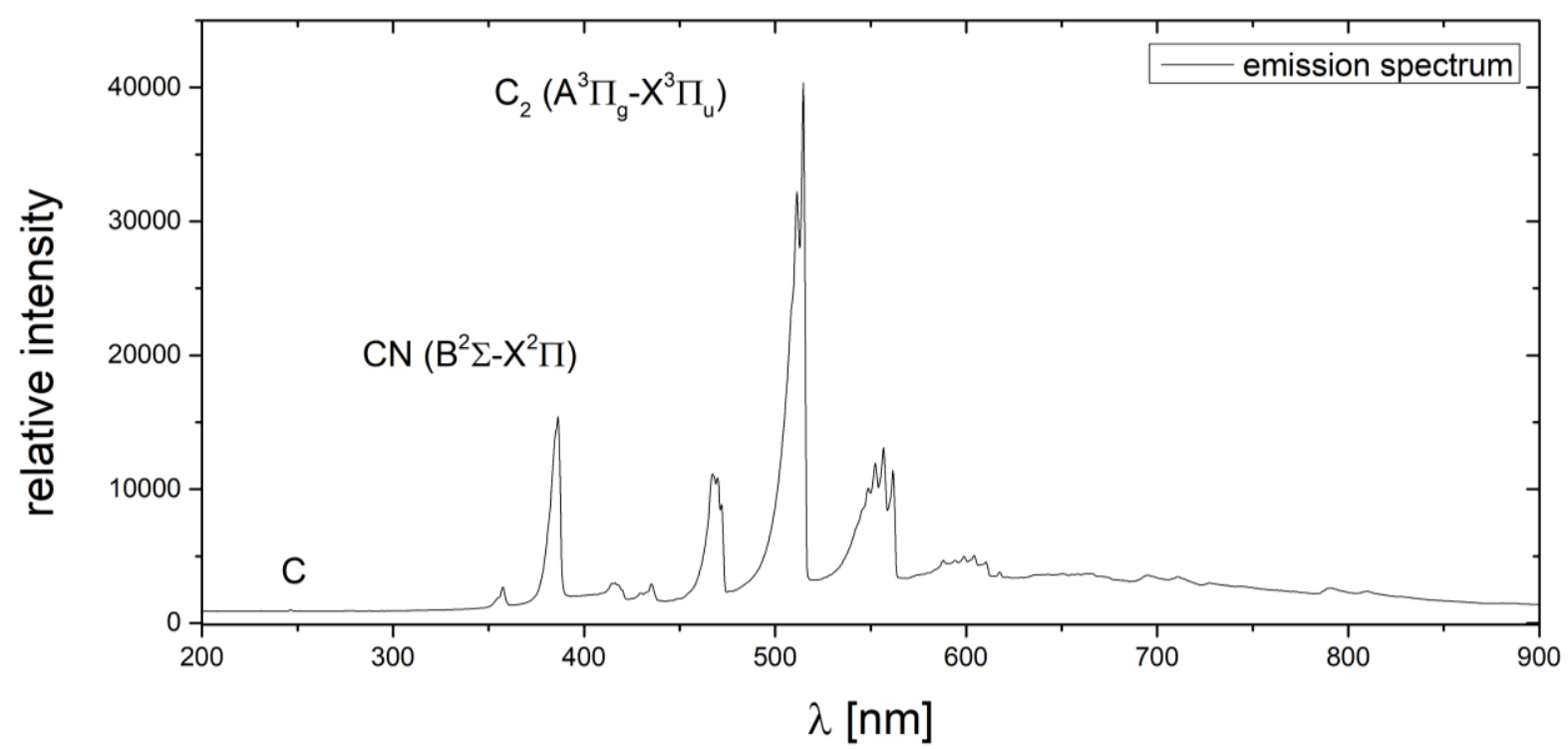

Figure 2 Overview emission spectra for $500 \mathrm{sccm}$ Ar (central), $700 \mathrm{sccm}$ Ar+EtOH (annular), $200 \mathrm{~W}$

Rotational structure of the violet $\mathrm{CN}$ system is often used for calculation of rotational temperature which can estimate the gas temperature in carbon containing plasmas $[3,7,8]$. This procedure can be done manually, or by using e.g. Lifbase [9] or MassiveOES [10] software. The gas temperature is an important parameter which strongly influences plasma chemistry and ethanol decomposition routes. Figure 3 (left) shows temperature distributions of the filament along the axis for two different MW powers.
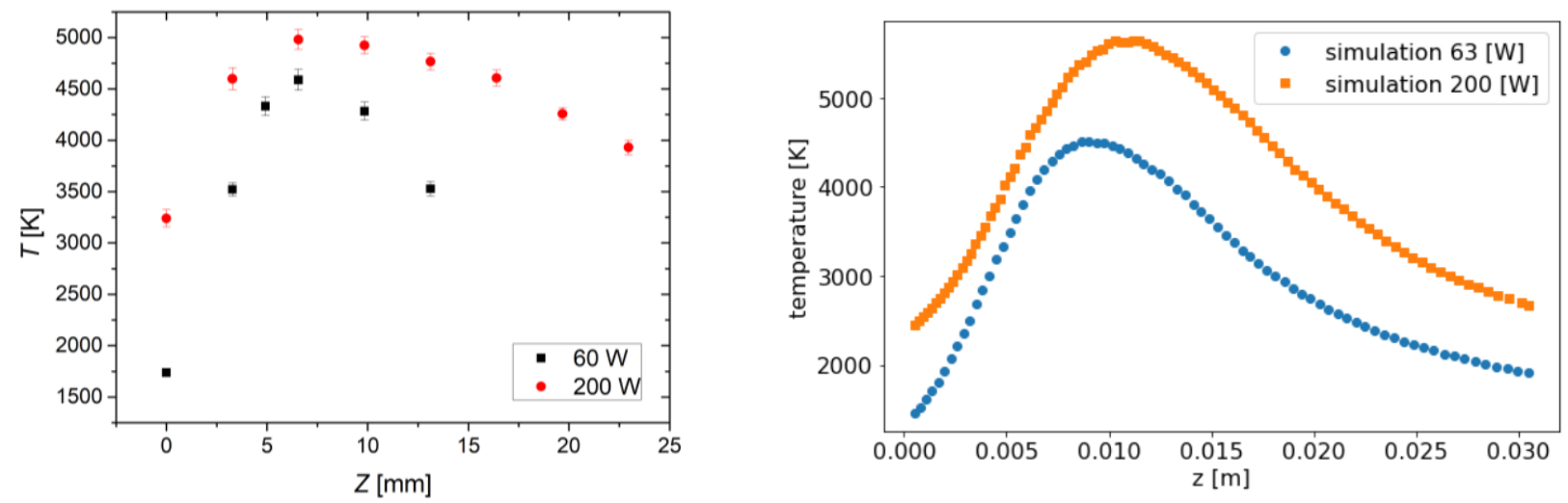

Figure 3 Gas temperature distribution - experimental (left (TROT (CN)), modelled (right) for $60 \mathrm{~W}$ a $200 \mathrm{~W}$, $500 \mathrm{sccm}$ Ar (axial), $700 \mathrm{sccm}$ Ar+EtOH (annular)

The theoretical model can provide better insight into plasma phenomena, producing, among others, spatial distribution of temperature or chemical species abundance. A comparison between experimental and modelled axial temperature profile is shown in Figure 3. Current model tends to underestimate the temperature further from the nozzle. This may be solved with right coupling of the energy transfer from excited argon metastables to the other species. 
While maximum temperature reached $4500 \mathrm{~K}$ threshold for effective formation of solid carbon [11] in both investigated conditions, higher microwave power fulfilled this condition in majority of its length, giving opportunity to formation of $\mathrm{C} 2$ and growth of planar structures over longer distance.

Analysis of exhaust gas showed that, as expected, the decomposition of ethanol was more complete for higher microwave power, see Figure 4. For lower microwave power (or high ethanol flow) the FTIR spectra are more complex as many intermediary species are present: methane, ethane, ethylene, acetylene, diacetylene, ethanol, $\mathrm{CO}, \mathrm{CO} 2, \mathrm{H} 2 \mathrm{O}, \mathrm{HCN}$. For higher microwave power (or low ethanol flow) only the acetylene, methane, ethylene, $\mathrm{CO}, \mathrm{CO} 2$ can be found in the FTIR spectrum. Overall, the main gaseous products of ethanol plasma decomposition are the acetylene and $\mathrm{CO}$.

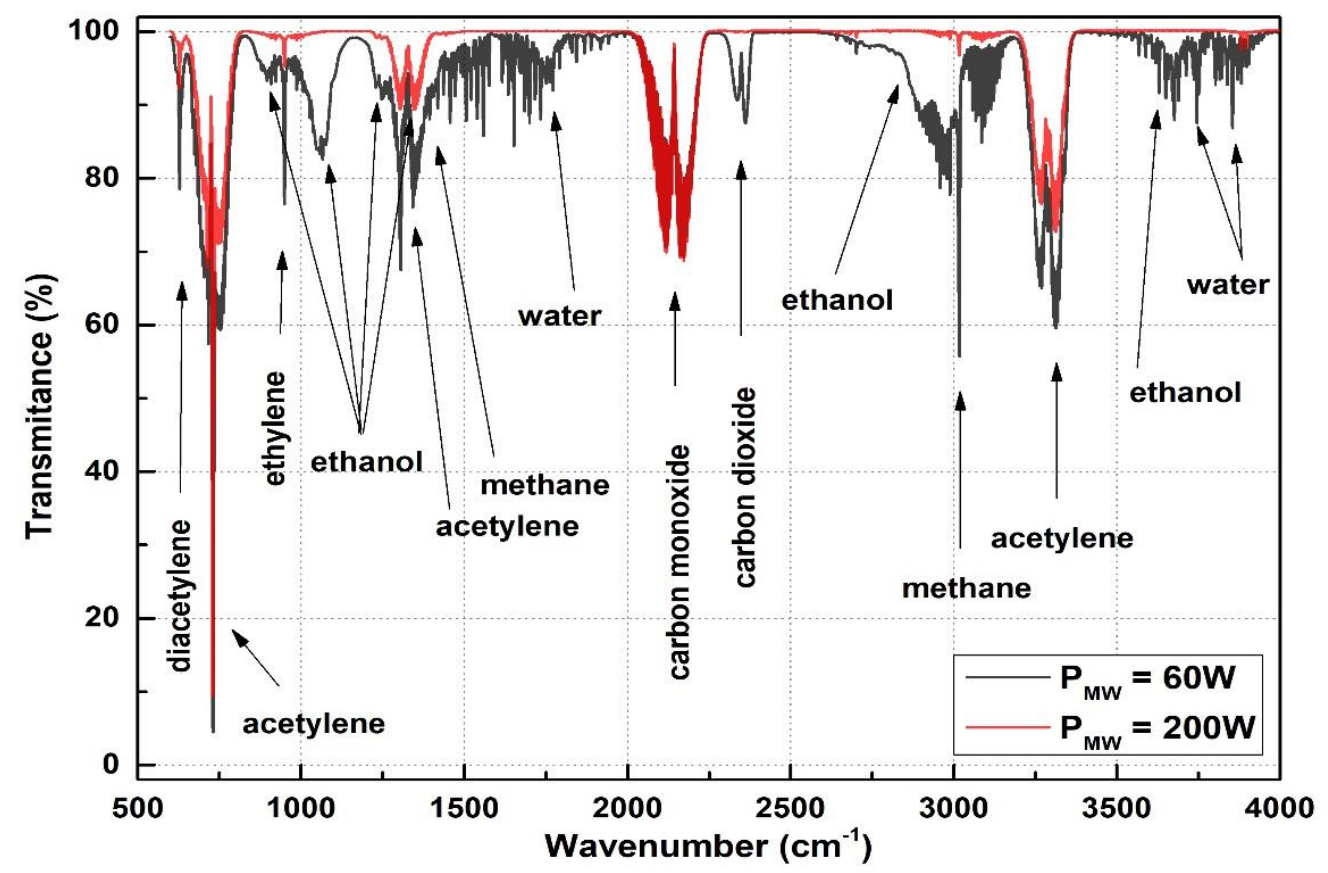

Figure 4 FTIR analysis of the exhaust gas for two microwave powers

The TOF MS spectra of the exhaust gas show a rich mixture of organic compounds with molecular weights mostly up to $132 \mathrm{amu}$. The repeating pattern with period of 26 amu suggests a significant role of $-\mathrm{C}_{2} \mathrm{H}_{2}$ - group. Acetylene line (mass $26 \mathrm{amu}$ ) is present, too, but its signal can be masked by $\mathrm{CN}$ with the same mass. The presence of $\mathrm{CN}$ in the gas is known from OES spectra taken from the active plasma region. The limited mass range of the spectrometer together with $1 \mathrm{~m}$ long sampling tube precluded the direct detection of the heavy carbon clusters of forming graphene.

SEM images show substantial differences in the structure of solid carbon nanomaterial produced at $60 \mathrm{~W}$ or $200 \mathrm{~W}$ of microwave power (Figure 5). At low power mode, lower gas temperatures are achieved leading to incomplete decomposition of ethanol resulting in the synthesis of spherical amorphous carbon nanoparticles instead of planar graphene nanosheets. Previous FTIR and TOF MS investigations provide important information about the gaseous products and an insight into the construction process. The simpler product structure dominated by $\mathrm{CO}$ (inevitable biproduct) and $\mathrm{C}_{2} \mathrm{H}_{2}$ (growth) at higher power homogenizes the growth leading to the hexagonal graphene lattice. The Raman spectroscopy (Figure 6) reveals a substantial difference in material properties. Intensive 2D peak together with relatively low $\mathrm{D}$ peak are evidence for few-layer graphene nanosheets with a relatively low amount of lattice defects. On the other hand, the reduced intensity of $2 \mathrm{D}$ peak and the broad and intensive $\mathrm{D}$ peak suggests presence of highly amorphous bulk material synthesized at lower microwave power. 

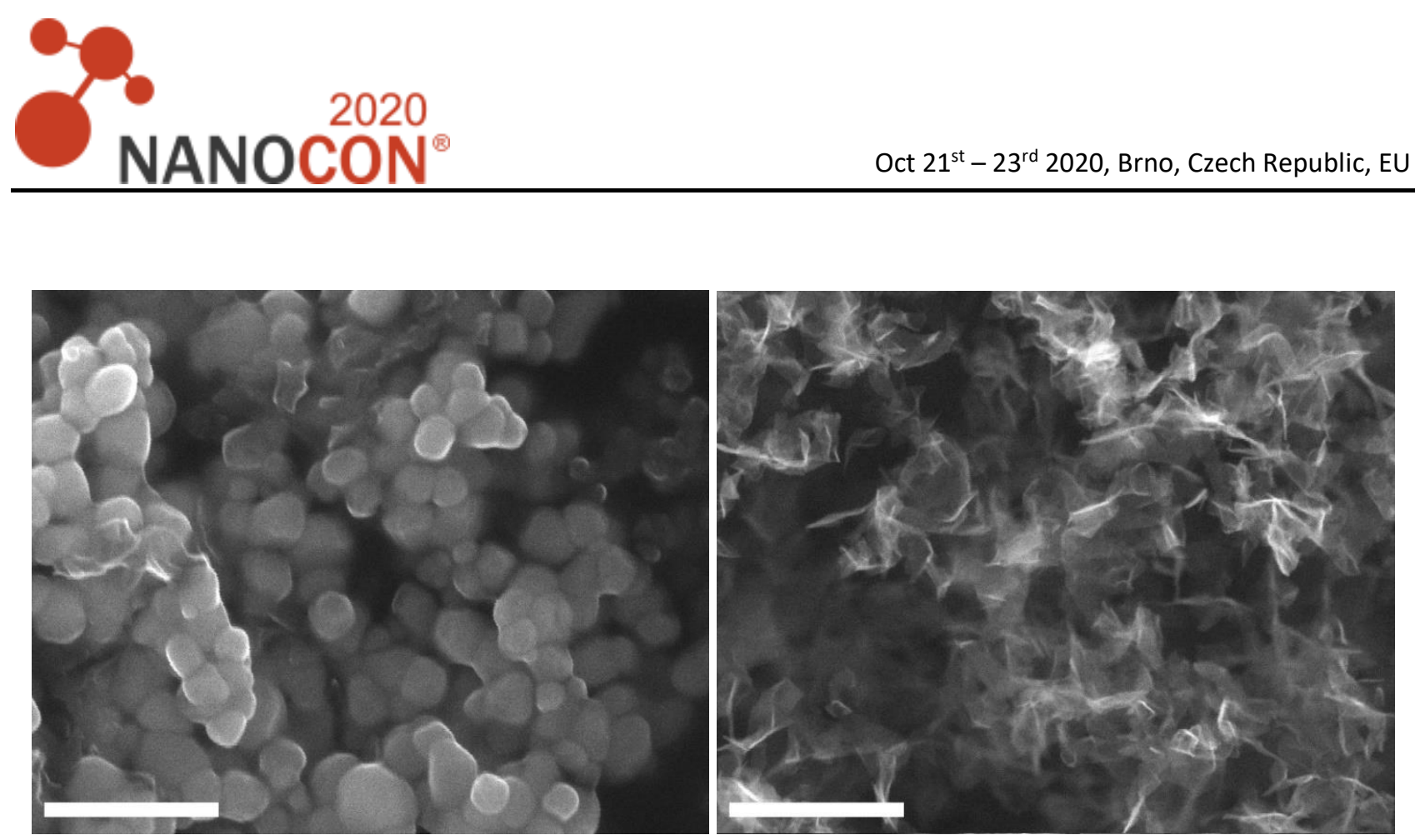

Figure 5 SEM image for $60 \mathrm{~W}$ (left) and $200 \mathrm{~W}$ (right), $500 \mathrm{sccm}$ Ar (central), $700 \mathrm{sccm}$ Ar+EtOH (annular). The scale bar represents $500 \mathrm{~nm}$
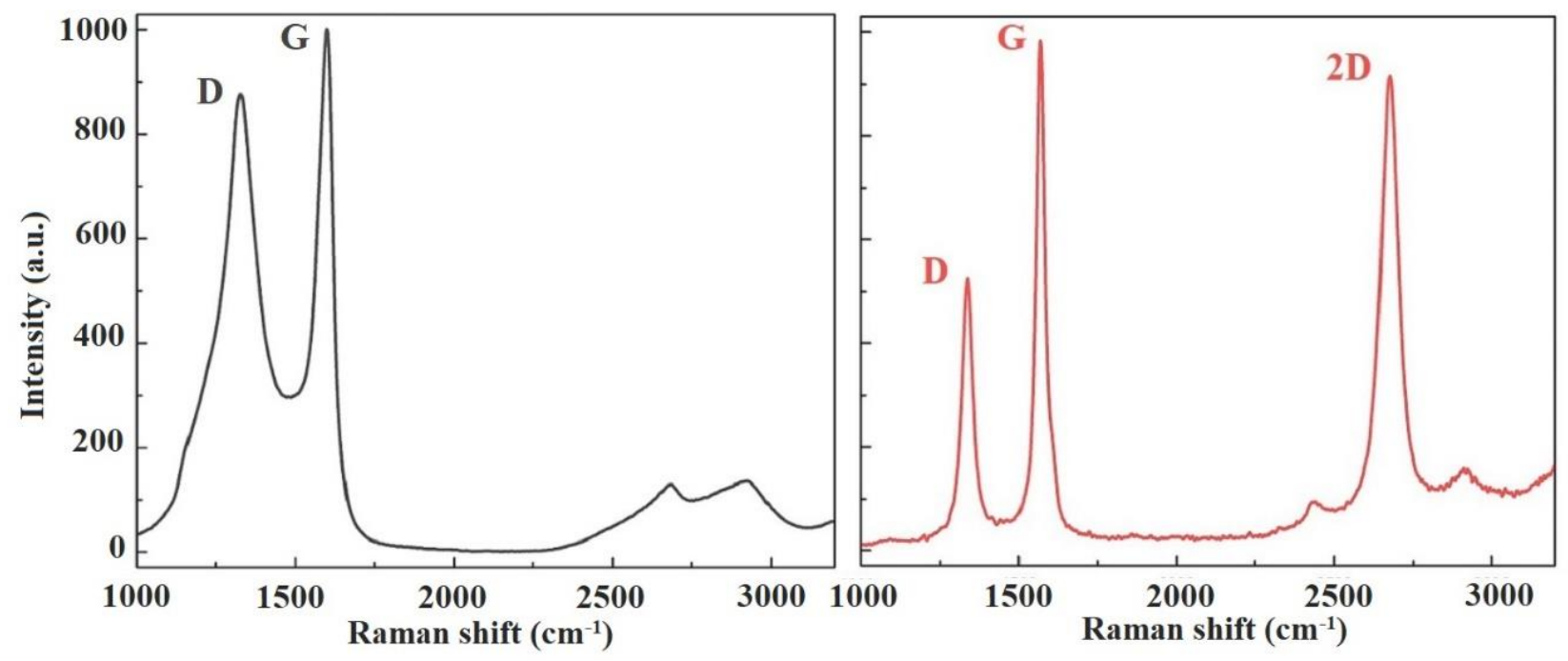

Figure 6 Raman spectra for $60 \mathrm{~W}$ (left) and $200 \mathrm{~W}$ (right), $500 \mathrm{sccm}$ Ar (central), $700 \mathrm{sccm}$ Ar+EtOH (annular)

\section{CONCLUSION}

Microwave power absorbed in the discharge can directly influence the gas temperature and gas kinetics during the synthesis of graphene nanosheets. Optical emission spectroscopy in conjunction with FTIR and mass spectrometry helped us identify the main species present in the process of ethanol decomposition under low and high plasma temperatures. The measured temperature profile of microwave discharge was in good agreement with modelling of plasma discharge under selected conditions showing maximum temperature in the main decomposition region $10 \mathrm{~mm}$ above the discharge nozzle. Nanosheets prepared in low power regime exhibited highly defective structure, high D/G Raman band ratio, in comparison to nanosheets prepared at a higher power, higher temperature regime. SEM images revealed structural change from amorphous nanoparticles to graphene which was directly related to generation of growth species $\left(\mathrm{C}_{2} \mathrm{H}_{2}, \mathrm{C}_{2}\right)$ as determined by OES and FTIR. 


\section{ACKNOWLEDGEMENTS}

\section{This work was supported by The Czech Science Foundation under project 18-08520S and in part by project LM2018097 funded by the Ministry of Education, Youth and Sports of the Czech Republic.}

\section{REFERENCES}

[1] TATAROVA, E., et al. Microwave plasma based single step method for free standing graphene synthesis at atmospheric conditions. Applied Physics Letters. 2013, vol. 103, no. 13, p. 134101.

[2] MELERO, C., et al. Scalable graphene production from ethanol decomposition by microwave argon plasma torch. Plasma Physics and Controlled Fusion. 2016, vol. 60, p. 014009.

[3] TOMAN, J., et al. On the interplay between plasma discharge instability and formation of free-standing graphene nanosheets in a dual-channel microwave plasma torch at atmospheric pressure. Journal of Physics D: Applied Physics. 2019, vol. 52, no. 26, p. 265205.

[4] MERINO, A., et al. A multipurpose reduced mechanism for ethanol combustion. Combustion and Flame. 2018, vol. 193, pp. 112-122.

[5] MOISAN, M. et al. Contribution of surface-wave (SW) sustained plasma columns to the modeling of RF and microwave discharges with new insight into some of their features. A survey of other types of SW discharges. Plasma Sources Science and Technology. 2018, vol. 27, no. 7, p. 073001.

[6] TATAROVA, E., et al. Microwave plasmas applied for the synthesis of free standing graphene sheets. Journal of Physics D: Applied Physics. 2014, vol. 47, p. 385501.

[7] NASSAR, H. CN violet system spectrum used as a molecular pyrometer and the influence noise to signal ratio on the temperature values. J. Phys. Conf. Ser. 2012, vol. 370, p. 012050.

[8] MOON, S. Y., et al. Temperature measurement of an atmospheric pressure arc discharge plasma jet using the diatomic CN (B $\Sigma+2-X \Sigma+2$, violet system) molecular spectra. J. Appl. Phys. 2009, vol. 105, p. 053307

[9] LUQUE, J., LIFBASE, S. software, Database and Spectral Simulation for Diatomic Molecules.

[10] VORÁČ, J., et al. Deducing Rotational Quantum-State Distributions from Overlapping Molecular Spectra. The Review of Scientific Instruments. 2019, vol. 90, no. 12, p. 123102.

[11] RINCÓN, R., et al. Experimental research on ethanol-chemistry decomposition routes in a microwave plasma torch for hydrogen production. Chemical Engineering Journal. 2016, vol. 284, pp. 1117-26. 